schen Vorgang der Dienstleistungserbringung herunterzubrechen und das Arzt-Patienten-Verhältnis im Sinne eines bloßen Erbringers und Nutzers von Leistungen in der Gesundheitsversorgung zu simplifizieren." Im Moment sei der Bereich der Organisationshaftung trotz seiner zunehmenden Bedeutung keiner systematischen Kontrolle zugänglich - weder bei Zivilgerichten noch bei der Überprüfung der Behandlungsdokumentation durch Gutachterkommissionen und Schlichtungsstellen, sagte Gaidzik weiter. Er verwies darauf, dass angesichts der immer besser werdenden diagnostischen und therapeutischen Maßnahmen die Risikobereiche ärztlichen Handelns nicht kleiner werden. „Gerade die immer stärker werdende Arbeitsteilung in den Kliniken, verstärkt durch den Zwang zur Wirtschaftlichkeit mit entsprechenden Konsequenzen für die Ausstattung und - last but not least - den Personalschlüssel, verstärkt die Tendenz, ärztliches oder pflegerisches Handeln als ,gefahrgeneigte Tätigkeiten' betrachten zu müssen.“

\section{Knackpunkt Dokumentation}

Obwohl diese Entwicklung nicht ohne Folgen für die Qualität der Versorgung bleibe, könne das deutsche Haftungsrecht mit dem Verbot des Ausforschungsbeweises nur begrenzt eingreifen. Der Grund: Organisationsdefizite im Klinikbetrieb spiegeln sich nur selten in der individuellen Behandlungsdokumentation des Patienten wider, erläuterte Gaidzik. „Mängel in der personellen Besetzung, Abteilungskoordination und nicht zuletzt -kommunikation oder auch in der Organisation der Rufbereitschaft werden in aller Regel nur per Zufall zu Tage treten." Der Patient werde kaum in Erfahrung bringen können, ob ein Behandlungsfehler das Resultat eines Konzentrationsmangels des übermüdeten Assistenzarztes oder seiner unguten Erfahrungen mit einem mehrfach aus dem Schlaf gerissenen Facharztes im Hintergrunddienst ist. „Um hier das Haftungsrecht wirkungsvoll als Instrument einzusetzen, bedürfte es neuer Wege im Prozessrecht", sagte Gaidzik.
Für die Zukunft hält es der Medizinrechtler auch für denkbar, dass sich die Gutachterkommissionen und Schlichtungsstellen im Bereich der Mediation engagieren könnten. Grundsätzlich entlastet die Arbeit der Kommissionen nach Einschätzung von Gaidzik nicht nur die Gerichte. Sie ist auch in der Lage, die dort durch den fehlenden medizinischen Diskurs bestehenden Defizite auszugleichen, „mit vielleicht sogar weit größerem Potenzial in der Zukunft“.

Die nordrheinische Gutachterkommission hat in den 40 Jahren ihres Bestehens mehr als 50.000 Anträge bearbeitet. Dabei haben die Ärzte und Juristen bei etwa einem Drittel einen Behandlungsfehler festgestellt. „Wiederholte Auswertungen der Ergebnisse haben nachgewiesen, dass es der Gutachterkommission in bis zu 90 $\%$ der Begutachtungsfälle gelingt, den Haftungsstreit zwischen Patienten und Ärzten durch das für die Beteiligten kostenfreie Begutachtungsverfahren beizulegen“, lobte der Vizepräsident der Ärztekammer Nordrhein Bernd Zimmer.

Ilse Schlingensiepen

\title{
Elvis, Goethe und der Da-Vinci-Roboter
}

\section{Ungewöhnlich ist neben dem 40-jährigen Praxisjubiläum vor allem die Historie, die sich hinter der ältesten urologischen Versorgungsstätte in Frankfurt am Main verbirgt.}

$\mathrm{m}$ m Jahr 1960 drehte Elvis Presley im Tanzcafé Europa auf der Zeil 123 in Frankfurt den Film „G.I. Blues“, in dem er seine berühmte Version von „Muß I denn zum Städele hinaus“ (Originaltitel: Wooden Heart) sang. Als das Café vor 40 Jahren für immer seine Pforten schloss, verlor Frankfurt eine der traditionsreichsten Institutionen dieser Art. Noch heute erzählen sich alte Frankfurter, wie sie auf der Treppe zum Café „Spalier saßen“ und der Musik lauschten, um sich den Eintrittspreis in das beliebte Etablissement zu sparen.

In der Folge wurde diese für viele Frankfurter historische Stätte in einer Pionierleistung zweckentfremdet: Auf mehr als 430 Quadratmetern gründeten die Urologen Doctores Klaus Peter Arndt und Knut Senger am 1. Juli 1975 die erste Gemeinschaftspraxis in der Mainmetropole überhaupt.

Im Dezember 2009 zog die Praxis aus dem inzwischen abgerissenen sogenannten Hako-Haus in die nur wenige hundert Meter entfernte Neue Kräme, die Johann Wolfgang von Goethe wohlwollend in "Dichtung und Wahrheit" erwähnte: „Der Römerberg war ein desto angenehmerer Spazierplatz. Der Weg nach der neuen Stadt, durch die Neue Kräme, war immer aufheiternd und ergetzlich.“

\section{Jahre, sechs "Generationen"}

Im Herzen Frankfurts, in unmittelbarer Nähe von Römer, Kaiserdom und der geschichtsträchtigen Paulskirche praktiziert inzwischen die sechste „Koalition“: Eva Hofmann, Dr. Mathias Eishold, Prof.
Dr. Ralf Bickeböller und Prof. Dr. Elmar Gerharz bieten das gesamte Spektrum der konservativen und operativen Urologie an. Seit dem 1. Februar 2012 gehört die Urologie an der Paulskirche zu dem Netzwerk UROGATE mit vier Standorten in Frankfurt und im Hochtaunus. Fünf der acht Urologen sind als Hochschullehrer an den Universitätskliniken in Frankfurt, Würzburg und Mainz in der Ausbildung von Medizinstudenten tätig.

Als im Jahr 2000 an der Universitätsklinik Frankfurt zum ersten Mal in der Geschichte der Medizin ein Da-VinciRoboter zur radikalen Entfernung der Prostata zum Einsatz kam, stammte der Patient aus der Praxis an der Zeil. Ein weiteres Ereignis, das die ungewöhnliche Historie der Gemeinschaftspraxis fortführt. Vielleicht hätte auch ein Mann wie Goethe die von der Deutschen Gesellschaft für Mann und Gesundheit e.V. als aktive Männergesundheitspraxis ausgezeichnete Praxis einmal aufgesucht, lebte er heute.

Susanne Brohm 